\title{
GRAPHITIC STRUCTURE AND SURFACE CHEMICAL ACTIVITY OF NANOSIZE CARBON SPHERES
}

\author{
Z. L. WANG* and Z. C. KANG \\ School of Materials Science and Engineering, Georgia Institute of Technology, Atlanta, GA 30332-0245, \\ U.S.A.
}

(Received 7 March 1996; accepted in revised form 31 October 1996)

\begin{abstract}
Monodispersed nanosize carbon spheres have recenlly been synthesized using a mixed-valent oxide-catalytic carbonization (MVOCC) process. In this paper, a high surface porosity of carbon spheres produced by an interaction with $\mathrm{WO}_{3}$ is reported. The interaction mechanism is due to the reduction of $\mathrm{WO}_{3}$ to $\mathrm{W}$ driven by an internal Auger decay process under the stimulation of a high-energy electron beam in a transmission electron microscope; combination of the desorbed $O$ with $C$ results in the loss of local carbon species. This process occurs only for the atoms located at the surface and surface diffusion plays a dominant role. Closed shell "onion-like" concentric fullerenes have been observed, with a $C_{60}$ molecule at the center. It is suggested that $W$ atom(s) may also be trapped within the $C_{60}$ cage. The spiral growth model proposed for the growth of large fullerenes and carbon spheres has been directly proved experimentally. High-resolution electron images suggest the presence of single-shell toroidal fullerenes. (C) 1997 Elsevier Science Ltd. All rights reserved
\end{abstract}

Key Words-A. Fullerenes, C. transmission electron microscopy, D. diffusion.

\section{INTRODUCTION}

The presence of carbon spheres in commercial carbon blacks has been known for a long time. Depending on the method of preparation, their diameter varies from 5 to $500 \mathrm{~nm}[1,2]$. The discovery of cagestructured fullerene molecules, $\mathrm{C}_{60}[3]$ and its family $\mathrm{C}_{n}[4,5]$ and carbon nanotubes [6] opened a new era of carbon research and applications. Carbon atoms can form pentagonal and heptagonal carbon rings, and the combination of these two basic structural units with the hexagonal carbon rings can form a variety of geometrical configurations, such as carbon spheres, cages, buckytubes [7], spiral tubes [8], etc. Fullerene carbon spheres with diameters of a few nanometers were first observed by Iijima [9] using high-resolution transmission electron microscopy (HRTEM). Carbon spheres were obtained in HRTEM by irradiating a soot containing tubular and polyhedral graphitic particles using a strong high-energy electron beam [10]. Heat treatment of pure carbon sool [11] and ultra-dispersed diamond powders $(2-6 \mathrm{~nm})$ [12] also produced small size carbon spheres. The carbon spheres are small and they tend to aggregate, and no technique has been found to disperse them. In the literature, these small size carbon spheres have been referred to as "onionlike" because the graphitic layers forming the particle are continuous and approximately closed shells.

Carbon spheres with sizes of $1-10 \mu \mathrm{m}$ were found in carbon black produced by carbonization of polyethylene-polyvinylchloride in a sealed gold tube under a pressure of $30 \mathrm{MPa}$ [13]. Carbon spheres

\footnotetext{
${ }^{*}$ Corresponding author
}

have also been found in carbon vapor produced by decomposition of $\beta$-SiC powder using conical converging shock-waves [14]. These techniques, however, are limited by several factors. First, the proportion of carbon spheres is low, and the spheres cannot be easily separated from the remaining carbon soot. For practical applications, a high percentage of pure carbon spheres is required. Second, the size distribution cannot be easily controlled, and the product is usually encapsulated on the surface of the catalyst. Finally, the carbon spheres may not be synthesized in macroscopic quantities as are needed for measurement of their physical properties and practical applications. Recently, a mixed-valent oxide-catalytic carbonization (MVOCC) process was found to yield monodispers carbon spheres at low cost $[15,16]$. The carbon spheres are formed by catalytic carbonization of natural gas with the assistance of a mixed-valent metal oxide. The carbon spheres formed are separated from the oxide catalyst, and no purification or further separation is needed. The product can be controlled by temperature to produce all spheres or tubes in macroscopic quantities [17]. The spheres are composed of portions of graphitic layers and arc expected to have high surface activity in catalysis, as demonstrated by the aggregation of the spheres after a simple ultrasonic treatment in acetone [18]. The microstructure and formation mechanism of carbon spheres have been described previously [19].

The chemical activity of the spheres is highlighted by the reaction of the $\mathrm{C}$ atoms on their surface with $\mathrm{WO}_{3}$ dispersed thereon. The porosity and reaction sites created by $\mathrm{WO}_{3}$ at the carbon surfaces are observed using high-resolution transmission electron microscopy (HRTEM), and the physical mechanism 
which drives this interaction process is illustrated. It is concluded that surface diffusion plays a dominant role. The carbon surface experiences a structural evolution, resulting in the formation of the onionlike carbon structures under the electron beam. The observation of spiral fullerenes supports the nucleation and initial spiral growth model proposed previously for carbon spheres [16].

\section{SPECIMEN PREPARATION}

Carbon spheres with an average diameter of $210 \mathrm{~nm}$ were synthesized using the MVOCC process [15]. Transition-metal oxides and/or rare earth metal oxides with mixed-valences are the catalysts. The spheres reported here were synthesized using $\mathrm{TbO}_{\mathrm{x}}$ as the catalyst. The carbon atoms form pentagonal, hexagonal and heptagonal carbon rings, the geometrical combination of these rings then form the carbon spheres/tubes. The MVOCC process is similar to the classical gas decomposition process under heating, but the key difference is the oxide catalyst. Many of the carbon electrode arc-discharge and the gas decomposition techniques reported in the literature both use metals, such as $\mathrm{Fe}, \mathrm{Ni}, \mathrm{Cu}$, transition metals or rare earth metals as catalysts. These catalysts not only promote carbonization of the gas but also provide nucleation sites for carbon products [8]. Thus, the catalysts are encapsulated after carbonization, and the purification of the final product is a major problem. The metal oxides with mixed valences used in MVOCC overcome this difficulty.

To study the surface chemical activity of these spheres, $\mathrm{WO}_{3}$ is ultrasonically dispersed in ethanol in a quantity sufficient to saturate the solvent and leave some of the oxide as a ultrafine suspension, enabling $\mathrm{WO}_{3}$ particles to adhere to the surfaces of the spheres, which were then picked up by a holey carbon grid for TEM observation. The HRTEM experiments were performed at $400 \mathrm{kV}$ using a JEOL 4000 EX TEM, which routinely gives a $0.18 \mathrm{~nm}$ image resolution.

\section{WO ${ }_{3}$ INDUCED SURFACE POROSITY}

The as-synthesized carbon spheres were first examined by scanning electron microscopy. The carbon spheres have an average diameter of $210 \mathrm{~nm}$. The full width at half maximum of the size distribution curve is $40 \mathrm{~nm}$. Figure 1(a) is a low-magnification TEM image of the as-synthesized spheres, which are clearly spherical. The carbon spheres are dispersed, clean, smooth and solid. An HRTEM image of the edge of a carbon sphere shows its graphitic structure. The graphitic flakes are not closed shells but small waving flakes of atom-thickness, ranging in sizes from $1-10 \mathrm{~nm}$. The arrangement of the graphitic flakes follows the curvature of the sphere at different depths. The interplane distance is modulated hy the wavy structure of the flakes, and the average

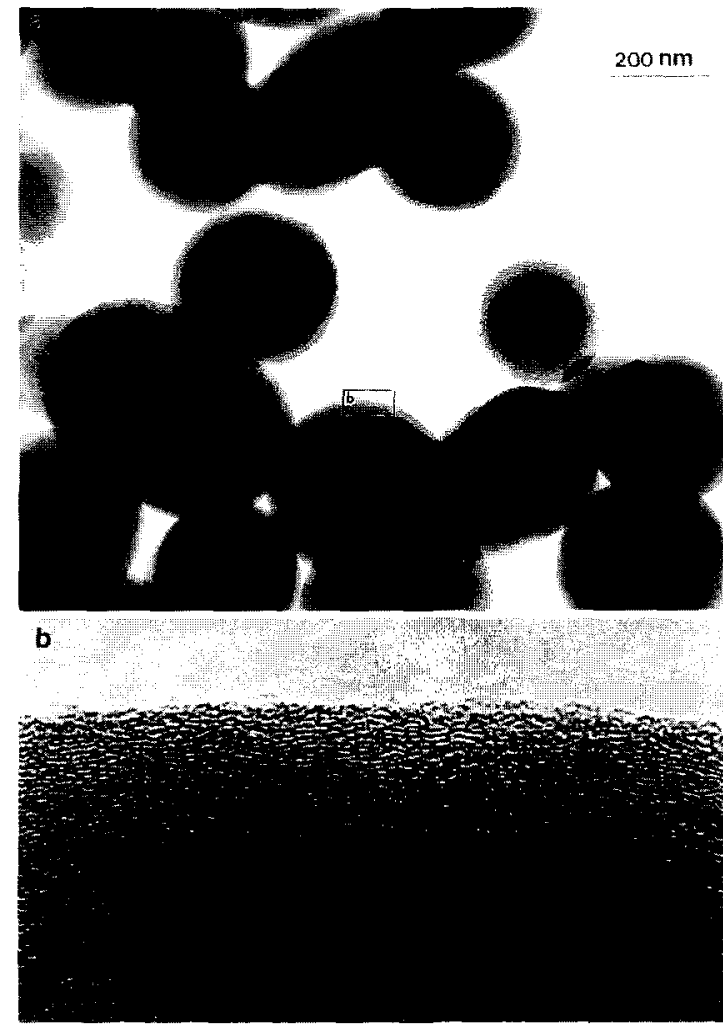

Fig. 1. (a) A low-magnification TEM image of the synthesized carbon spheres by the MVOCC technique. (b) An HRTEM image recorded from a region at the edge of a sphere as indicated in (a) showing wavy graphitic layered structures, but each layer is an unclosed shell and composed of many flakes.

interplane distance between the flakes is approximately $0.34 \mathrm{~nm}$. The unclosed graphitic flakes create many open edges, which are highly desirable to activate surface reactions. These open edges have been shown to be the key microstructure responsible for the aggregation between spheres after an ultrasonic treatment in acetone [18]

The first structure for particles found in carbon black was proposed as a hollow shell polyhedron [19]. Later, this structure was changed by Heidenreich et al. [20] to that of a hollow spherical shell composed of piles of hexagonal graphite flakes. with radial $c$-axes so that the layer planes were tangential to the particle surface. The key difference between this model and the growth model of the carbon spheres synthesized by MVOCC is that the pentagonal and heptagonal carbon rings are introduced in our model according to HRTEM results $[16,17]$, as summarized below.

A carbon sphere is believed to be nucleated from a pentagonal carbon ring followed by a spiral shell growth. This is the growth mechanism first proposed for the formation of large carbon fullerenes [4]. This growth core is believed to be formed in or rather on the solid phase under the action of the oxygen in the lattice of the catalyst, after which the sphere, once 
formed, separates from the catalyst. The lattice oxygen provided by the catalyst under heating is required to oxidize $\mathrm{CH}_{4}$. The spiral growth is the key for the nucleation of the sphere but it is not adequate for growing large spheres because of the limited growth rate along the spiral direction [16]. When the sphere grows larger, graphitic flakes of atom-thickness are nucleated on the surface due to the arrival of the pentagonal, hexagonal and heptagonal carbon rings. A flat graphite layer is composed of hexagonal carbon rings. The presence of a pentagonal carbon ring in a previously plane assembly of hexagonal carbon rings creates a structure with a local positive Gaussian curvature, whereas the presence of a heptagonal ring results in the formation of a local saddleshaped defect of negative Gaussian curvature [21]. While observed in TEM, the projections of these structures along the incident beam direction give a fringe curved inward or outward and the bending angle depends on the projection direction of these 3-D objects. The pentagonal, hexagonal and heptagonal carbon rings are required to modify the graphitic flakes for accommodating the local curvature of the carbon sphere. Geometrical combinations of these carbon rings can make all the structural configurations required to accommodate the surface curvature. Therefore, the spheres are composed of curled graphitic flakes with random twisting because there is almost no correlation among the graphitic flakes, and the growth temperature $\left(1100^{\circ} \mathrm{C}\right)$ is not high enough to improve the local order.

The graphitic flake structure of the spheres is expected to have chemical characteristics very different from those with onion-like structure $[10,11,22]$ with closed shell structures. The closed shell structure is likely to be chemically inert because the energy required to break the in-plane covalent bonds of graphite is high and the carbon atoms located in the graphitic planes have no dangling bonds to combine with other atoms. Foreign atoms are expected to be adsorbed only at open edges of the graphitic layers. In fact, the carbon spheres with closed shell structure are not expected to be formed in the MVOCC process because of high nucleation rates of the pentagonal and heptagonal carbon rings in the growth chamber. The carbon onions may not grow into large spheres because the atom species must fall on the edge of the in-plane growth front in order to continue the spiral growth. In practice, many graphitic flakes are nucleated simultaneously on the surface and there might be no correlation among their orientations. Thus, the unclosed graphitic layers are formed layer-by-layer. This is the model for growing large spheres.

In order to examine the surface chemical activity of the carbon spheres, nanosize $\mathrm{WO}_{3}$ particles are suspended in ethanol in which carbon spheres are dispersed. A low-magnification TEM image of a
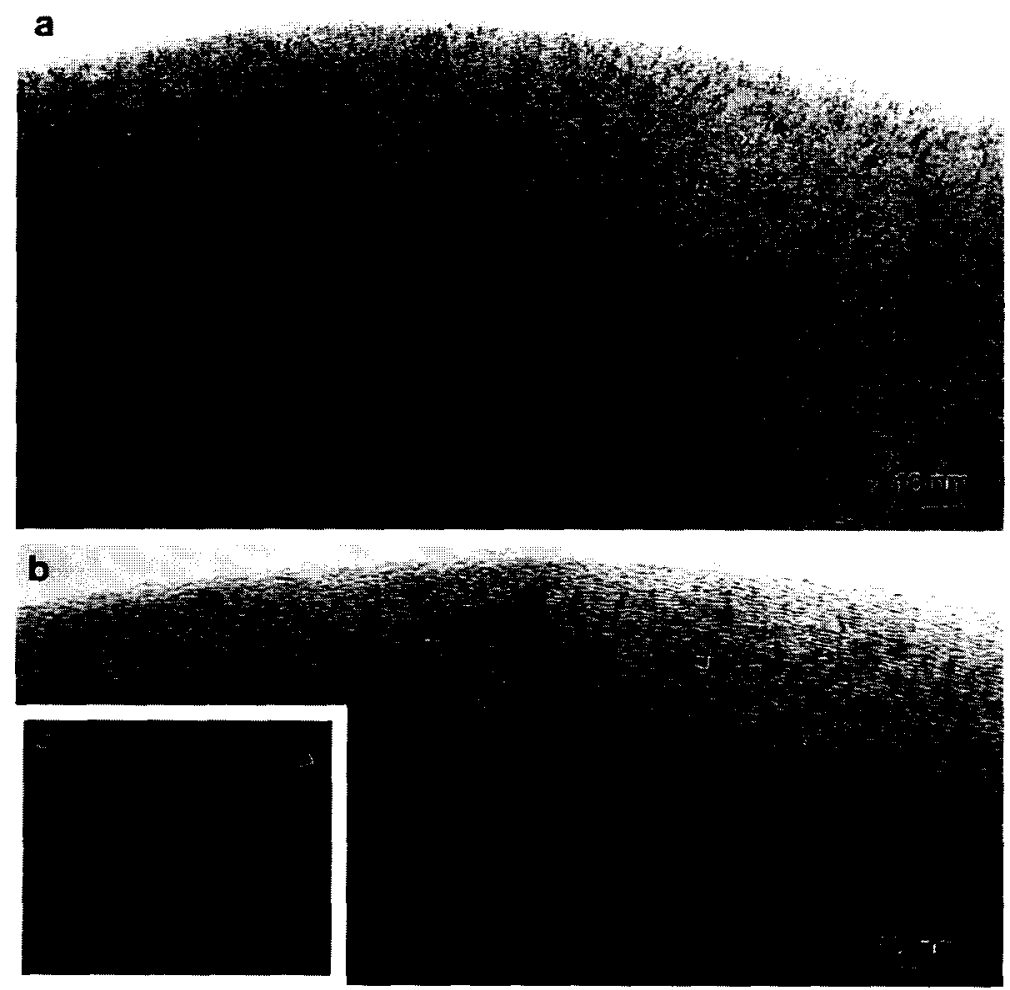

Fig. 2. (a, b) Low-magnification TEM images of carbon spheres showing small $\mathrm{WO}_{3}$ clusters dispersed on the surfaces. (c) An HRTEM image showing that the $\mathrm{WO}_{3}$ clusters are crystalline. 


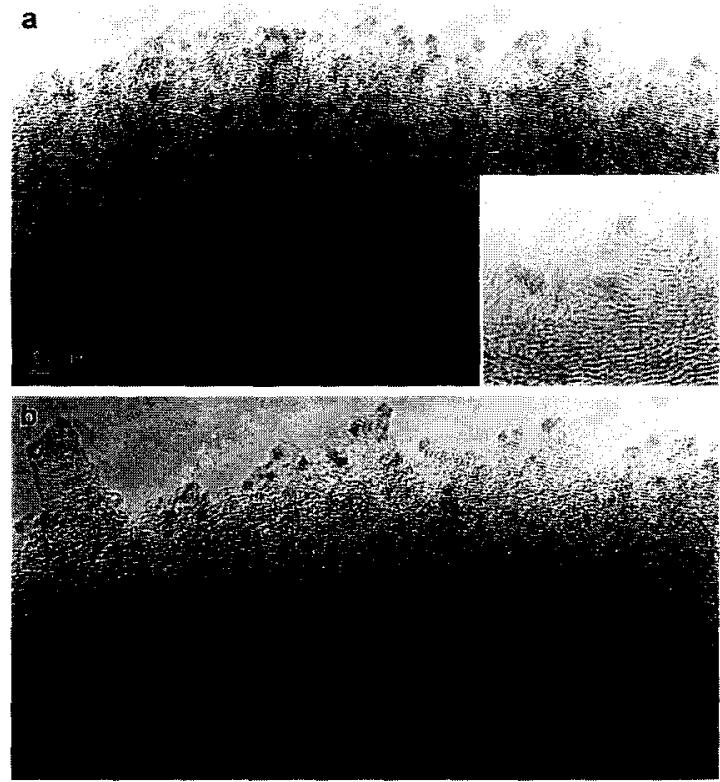

Fig. 3. HRTEM images showing the surface porosity produced by the "etching" effect of the $\mathrm{WO}_{3}$ clusters under the stimulation of a high-energy electron beam
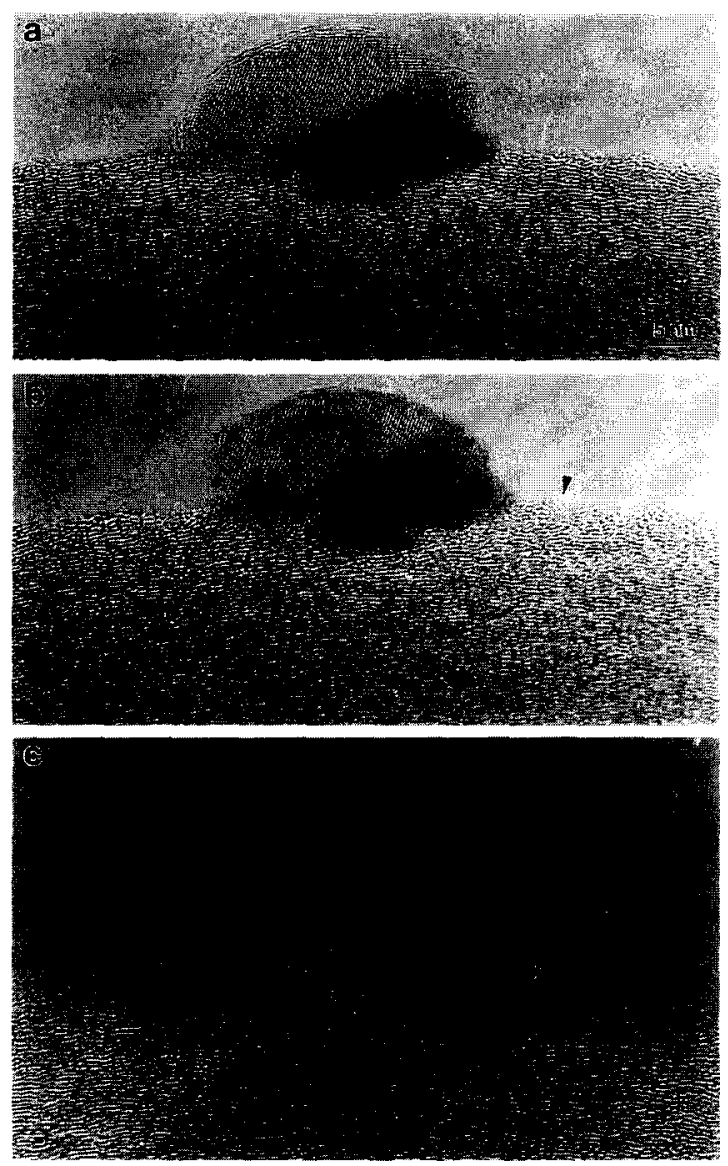

Fig. 4. Interaction of an $\mathrm{WO}_{3}$ particle with the surface of a carbon sphere, showing the loss of carbon at regions near the particle but not the region beneath the particle, resulting in the formation of a neck structure. carbon sphere clearly indicates the decoration of small $\mathrm{WO}_{3}$ clusters, in sizes as small as $0.5 \mathrm{~nm}$, uniformly distributed on the carbon surface (Fig. 2(a)). The clusters, which are better seen in Fig. 2(b), are crystalline, as indicated by the lattice fringes in Fig. 2(c). The average distance between adjacent clusters is approximately $3 \mathrm{~nm}$. For a carbon sphere of $200 \mathrm{~nm}$ in diameter, there are more than $10000 \mathrm{WO}_{3}$ clusters on its surface. These small size clusters are believed to be formed in the dissolution process of $\mathrm{WO}_{3}$ in ethanol, and they tend to distribute at the sites having open graphitic edges on the surface of the carbon sphere. This is a useful technique for producing ultratine $\mathrm{WO}_{3}$ clusters.

The adhesion of $\mathrm{WO}_{3}$ clusters on the surface greatly changes the structure of the carbon spheres under the stimulation of the electron beam. Figure 3 shows two HRTEM images of the edges of carbon spheres, where many $\mathrm{WO}_{3}$ clusters are seen. In comparison to the image shown in Fig. 1(b), the carbon surfaces shown in Fig. 3 appear to have strong interaction with the $\mathrm{WO}_{3}$ clusters under the electron beam, resulting in high surface porosity. It is also noticeable that the $\mathrm{WO}_{3}$ clusters are located at the top of the surface roughness created. This occurs even for a large $\mathrm{WO}_{3}$ particle (Fig. 4). Under a strong electron beam, carbon surface erosion starts at the regions adjacent to the particle (Fig. 4(b)). After about 30 minutes under the electron beam, the surface morphology is completely changed and many hills and valleys have been created. A neck is formed between the sphere and the particle, and this region is least affected by the mass loss (Fig. 4(c)). This interaction process creates many open graphitic edges at the carbon surface and the surface area is thus greatly increased. This is useful in catalytic applications.

The evolution of the structures in Figs 2 and 3 suggest that the interaction between the $\mathrm{WO}_{3}$ clusters and the carbon sphere occurs via surface diffusion rather than direct contact. This can be interpreted using an internal Auger decay mechanism proposed by Feibelman and Knoteck [23] for interpreting the electron stimulated desorption of $\mathrm{WO}_{3}$. Under the excitation of the electron beam, one electron in the W4f or W5p state is ionized, after which one O2p electron fills the hole created by the ionized $W$ atom; the energy released from this down transition is sufficient to ionize two $\mathrm{O} 2 \mathrm{p}$ electrons. Thus, $\mathrm{O}^{2-}$ loses up to three electrons and is thus converted into a positively charged $\mathrm{O}^{+}$ion. Since $\mathrm{WO}_{3}$ is dominated by ionic bonding, which is $\mathrm{W}^{+6} \mathrm{O}_{3}^{2-}$, the Coulomb repulsion between $\mathrm{O}^{+}$and an $\mathrm{W}^{+6}$ results in the desorption of $\mathrm{O}^{+}$, and $\mathrm{WO}_{3}$ is reduced to $\mathrm{W}$. Since an $\mathrm{O}^{+}$ion located beneath the surface layer is unlikely to escape, this desorption process occurs only for the atoms located at the surface. The wandering $\mathrm{O}^{+}$ions tend to be attracted to the $\mathrm{p}$ electrons of the carbon atoms located at the edges of the open graphitic layers on the carbon surface. The combination of $\mathrm{O}$ with $\mathrm{C}$ produces gaseous $\mathrm{CO}_{2}$ or $\mathrm{CO}$, 
resulting in the loss of local carbon and the formation of surface porosity. The individual $\mathrm{W}$ atoms may tend to diffuse to the surface of the carbon sphere, where they aggregate to form the clusters observed (Fig. 3). This phenomenon was used by Iijima [24] to prepare ultrathin graphite substrates for imaging single $\mathrm{W}$ atoms using HRTEM. It must be pointed out that this process is likely to occur for atoms originating from the surface $\mathrm{WO}_{3}$, thus the reaction occurs in the vicinity of the $\mathrm{WO}_{3}$ particle rather than at the contact region between $\mathrm{WO}_{3}$ and the carbon sphere because the $O$ ions located within the contact region cannot escape, resulting in the formation of the neck structure observed in Fig. 4(c).

Because a significant fraction of carbon atoms have been removed, a large quantity of oxygen atoms must have been required to make this oxidation possible, but a small shrinkage in the size of the $\mathrm{WO}_{3}$ particle is not sufficient to account for the oxygen needed for the reaction. The remaining oxygen could have come from the nanoclusters dispersed on the sphere surface (see Fig. 2). It is also likely that the $\mathrm{WO}_{3}$ crystal is reduced to $\mathrm{W}$ so that the oxygen ions diffuse to the surface and then combine with carbon to form $\mathrm{CO}$ or $\mathrm{CO}_{2}$, resulting in a loss of carbon. On the other hand, the loss of oxygen in $\mathrm{WO}_{3}$ does not introduce any significant change in crystal volume.

\section{CONCENTRIC AND TOROIDAL FULLERENES}

As pointed out earlier, the interaction of $\mathrm{WO}_{3}$ clusters with the carbon surface creates many open edges at that surface and the graphitic flakes located at the open edge regions would tend to curl to form large fullerenes. Figure 5(a) shows an initial formation stage of a fullerene type of structure, and a dip region created at the surface. After an extended period of electron beam illumination, many curled graphitic layers are formed on the surface (Fig. 5(b)). The graphitic layers with sharp turning angles are seen in the area indicated by an arrowhead in Fig. 5(b), possibly corresponding to the presence of pentagonal carbon rings or $\mathrm{sp}^{3}$-hybridized $\mathrm{C}$ atoms. The pentagons cause the graphitic layers to curl, resulting in the spiral growth of onion-like fullerenes (Fig. 5(c)) [4].

Many closed shell structures have been seen, and these fullerenes are based on $\mathrm{C}_{60}$, with a diameter of $0.7 \mathrm{~nm}$ directly measured from the HRTEM image (Fig. 6). A correct understanding of image contrast dependence on the defocus of the objective lens in TEM is important for identifying the existence of $\mathrm{C}_{60}$ within the onion-like sphere, because contrast reversal can be introduced by a change of lens defocus, which is a typical characteristic of phase contrast imaging in HRTEM [25]. In Fig. 6, the bright contrast fringes correspond to the projection of the graphitic layers, thus the center of the $\mathrm{C}_{60}$ is cxpected to exhibit dark contrast if there is/are no other atom(s) trapped inside the cage. However, the center of the $\mathrm{C}_{60}$ in region 2 shows bright contrast, indicating that one or more $\mathrm{W}$ atoms might be trapped inside the cage. This is likely to be the case because the small size clusters of $\mathrm{WO}_{3}$ were initially dispersed on the surface (see Fig. 2), and it is possible to reduce them to single $\mathrm{W}$ atoms under the electron beam [24]. Moreover, the onion-like structure is rarely formed on the surface of a clean carbon sphere without a decoration of $\mathrm{WO}_{3}$ according to our previous observations [16]. Thus, it is suggested that the $\mathrm{W}$ atom(s) is/are likely to be wrapped up by a graphitic flake with the presence of pentagons. The $\mathrm{C}_{60}$ will be wrapped up by a graphitic flake with larger size, resulting in the formation of concentric fullerenes. The electron beam is needed not only for reducing $\mathrm{WO}_{3}$ to $\mathrm{W}$ atoms but also for providing the activation energy needed for the wrapping process. The closed shell structure is concentric $\mathrm{C}_{60} @ \mathrm{C}_{240}$ (with diameters of 0.7 and $1.4 \mathrm{~nm}$, respectively).

Many unclosed spiral-shell structures have been observed (Fig. 7). The spiral laycrs are sharply bent at the beginning of the growth due to the formation of pentagonal carbon rings (or $\mathrm{sp}^{3}$-hybridized $\mathrm{C}$ atoms) at the corner. The size of the first spiral is approximately $0.7 \mathrm{~nm}$, in correspondence to the diameter of a $\mathrm{C}_{60}$ molecule, and the particle has a polyhedral shape. These images provide strong evidence supporting the spiral growth model proposed for the formation of large fullerenes [4] and the nucleation and initial growth of the carbon spheres [14]. It also suggests that the pentagons are the nucleation sources for the spiral growth.

Geometrical combinations of hexagonal, pentagonal and heptagonal carbon rings can produce various shapes. The positive and negative curvatures created by pentagons and heptagons [21], respectively, can force the graphitic layers to match any desired surface geometry. Various aspects of such pentagonal and heptagonal carbon ring pairs have been discussed from a theoretical point of view $[26,27]$. Many singleshell graphitic carbon fullerenes have heen observed in HRTEM images, as shown in Fig. 8. The fullerene at region 1 has a length about $2 \mathrm{~nm}$ and a height of $0.4 \mathrm{~nm}$. This type shape may correspond to the singleshell toroidal fullerenes predicted theoretically by Ihara and Itoh [28], who pointed out that the smallest height of a torus fullerene is $0.37 \mathrm{~nm}$, in agreement with the structure observed in region 1 , but the inner wall is not clearly seen possibly because of its small size. The graphitic structure at region 2 has a dimension of approximately $0.7 \mathrm{~nm}$, in correspondence to the diameter of a hemisphere of $\mathrm{C}_{60}$ which is the smallest cap in carbon nanotubes [29-31]. For the single-shell torus structures, a flat graphitic layer can be introduced inside the cage if the height of a torus is larger than $0.68 \mathrm{~nm}$ because the interlayer distance is $0.34 \mathrm{~nm}$. This is just shown by the observation in region 2 . Region 3 has a similar structure, but it seems that the fullerene has a football shape. The fullerene at region 4 may be an unclosed structure. 

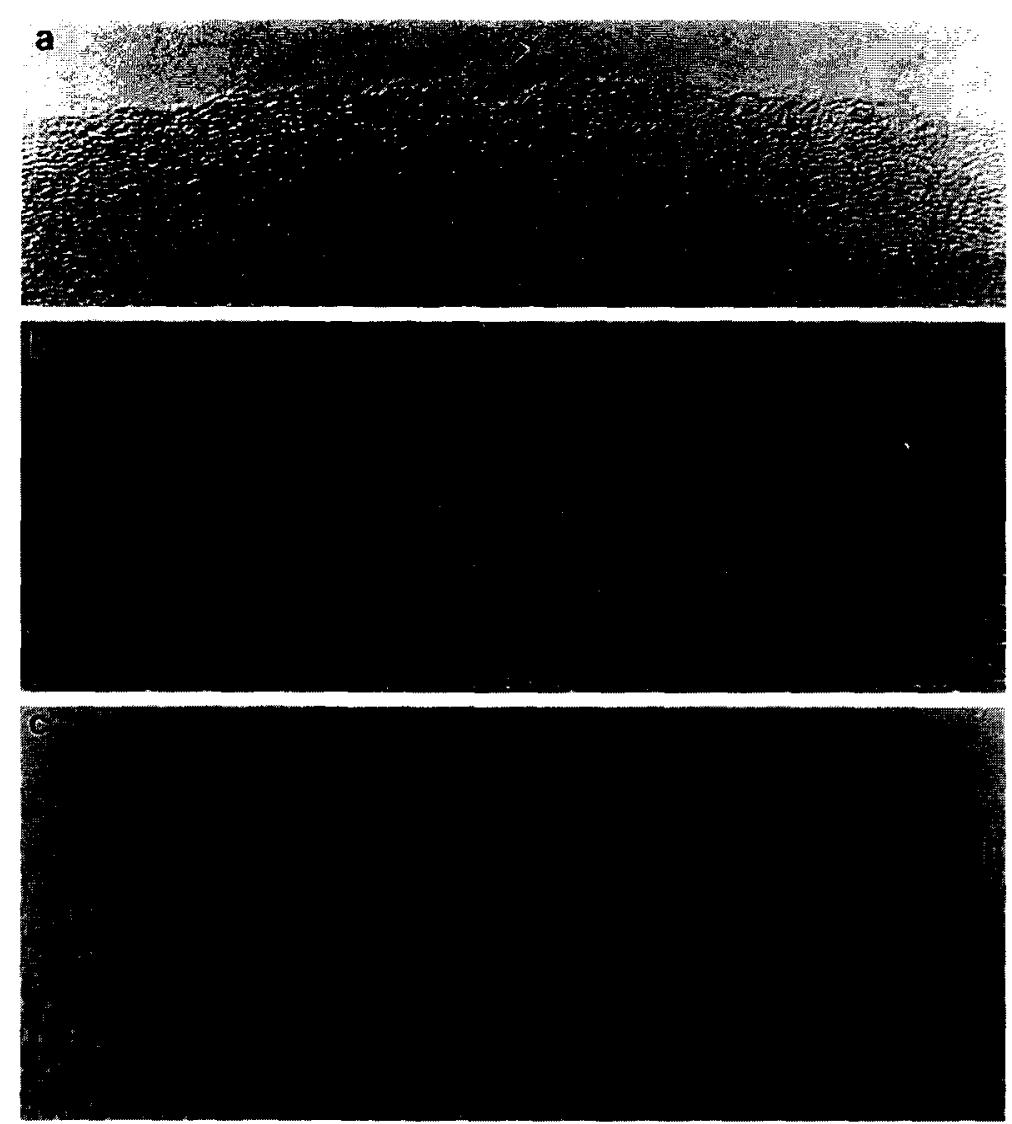

Fig. 5. Growth of curled graphitic structures at the sphere surface with the promotion of W atoms/clusters and the stimulation of the electron beam.

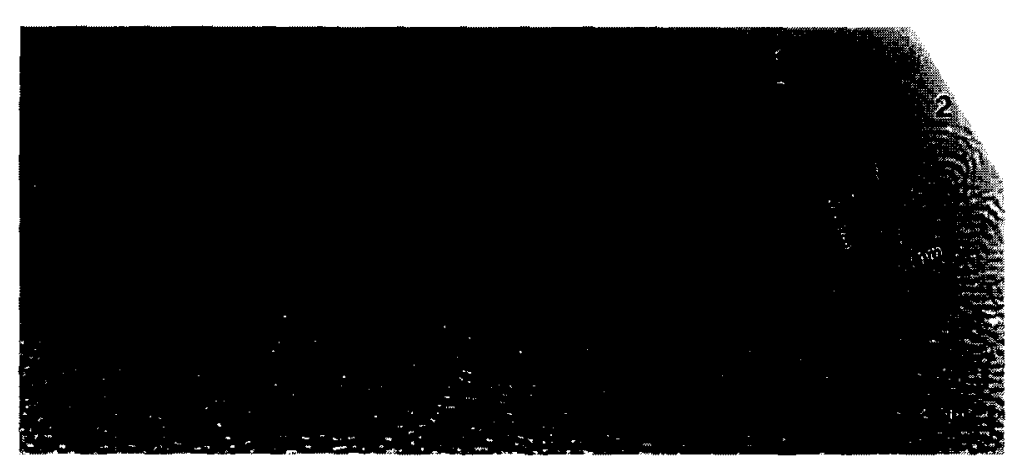

Fig. 6. An HRTEM image showing the formation of closed-shell fullerene structures at the sphere surface (see text). The innermost fullerene is $\mathrm{C}_{60}$, and one or more $\mathrm{W}$ atoms is/are likely to be trapped at the center.

All these observations have proved a fact that a combination of hexagonal, pentagonal and heptagonal carbon rings can produce a variety of geometrical shapes. This is one of the magic characteristics of carbon.

\section{CONCLUSIONS}

In this paper, HRTEM observations are reported on the interaction between $\mathrm{WO}_{3}$ clusters and carbon spheres synthesized using a mixed-valent oxide-catalytic carbonization (MVOCC) process. Many subna- nometer $\mathrm{WO}_{3}$ clusters can be uniformly dispersed on the surfaces of the carbon spheres. The carbon spheres are attacked by $\mathrm{WO}_{3}$ under an electron beam and a high porosity is created at the surface. The surface area and the density of reaction sites are greatly increased. The interaction mechanism is believed due to the reduction of $\mathrm{WO}_{3}$ to $\mathrm{W}$ atoms as a result of an internal Auger decay process under the stimulation of an electron beam in TEM, and a combination of the desorbed $\mathrm{O}$ with $\mathrm{C}$ results in the loss of local carbon species, forming the porous structure. This interaction occurs only for the atoms 

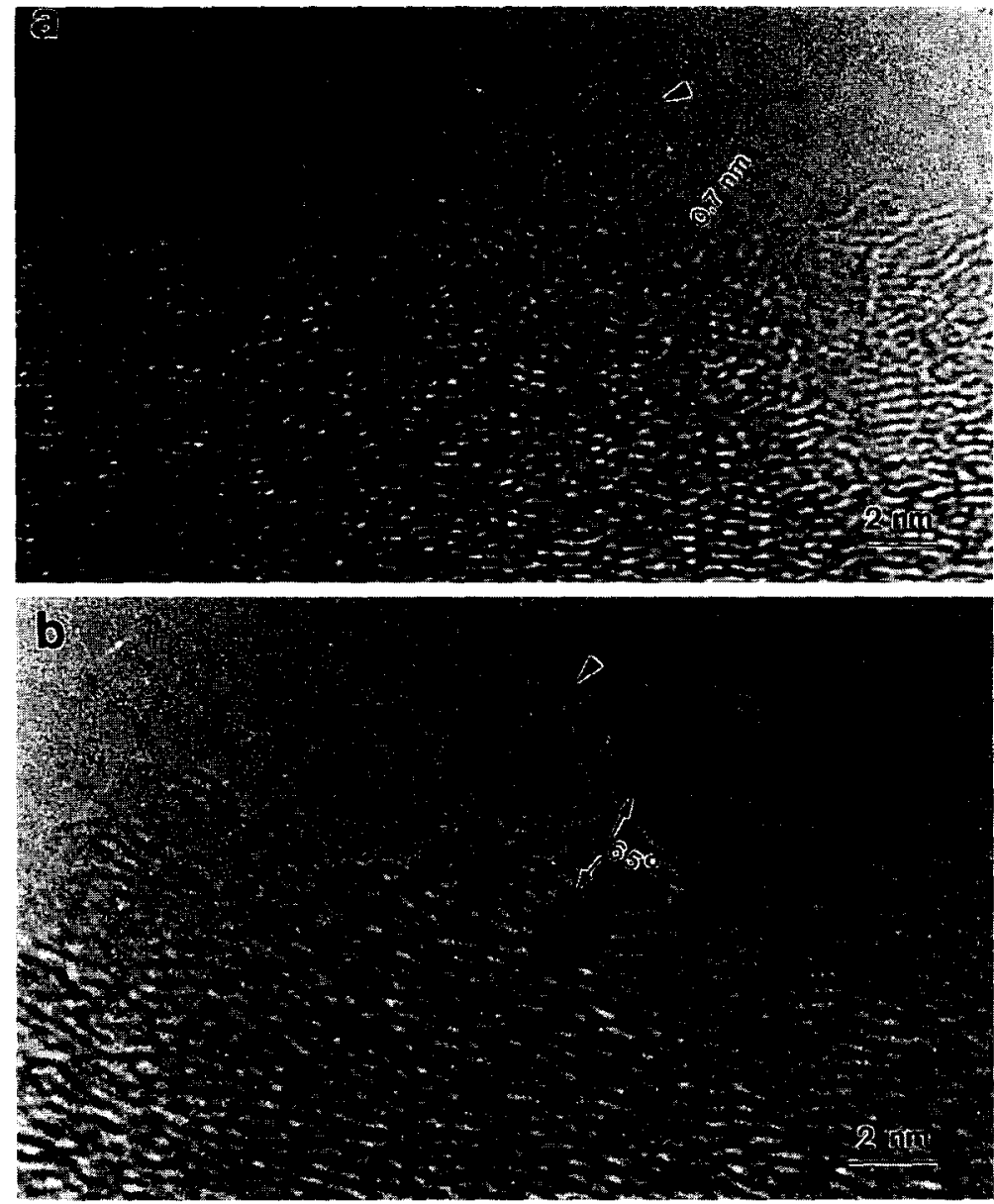

Fig. 7. HRTEM images showing the formation of unclosed spiral structures at the sphere surface. The spiral structure is proposed as the nucleation and initial growth model of the synthesized large carbon spheres.
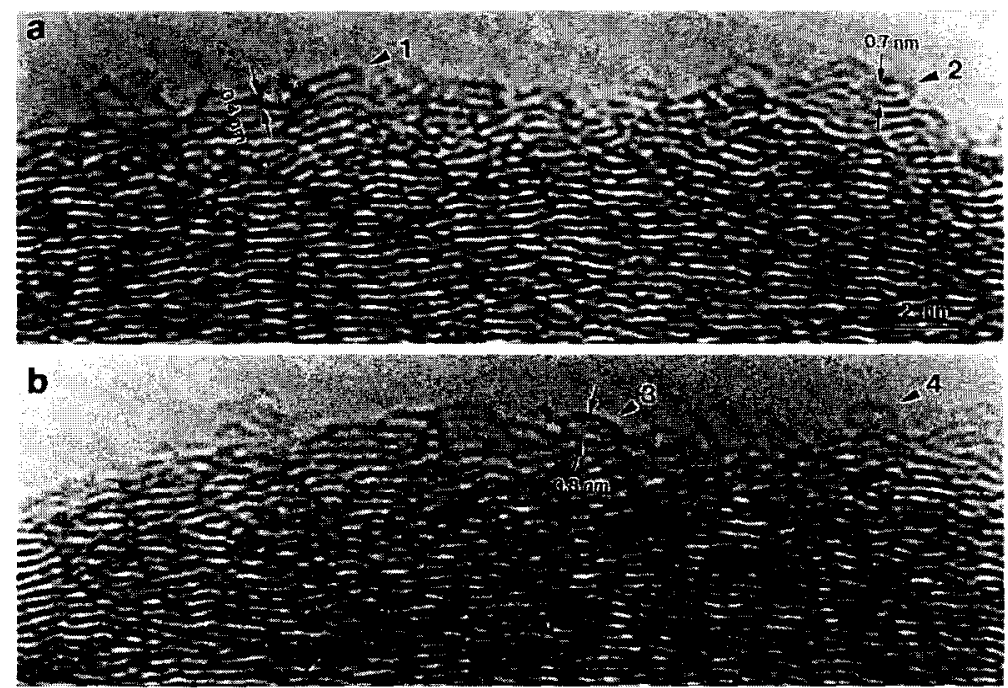

Fig. 8. Direct observation of single-shell toroidal fullerenes by HRTEM at the sphere surface. These images provide the first evidence of the torus fullerenes predicted theoretically by Ihara and Itoh [28].

located at the surface rather than those at the contact area between the sphere and the $\mathrm{WO}_{3}$ particle because the $\mathrm{O}$ ions inside the particle cannot escape.
Closed shell "onion-like" concentric fullerenes have been observed, with a $C_{60}$ molecule at the center. It is suggested that the $\mathrm{W}$ atom(s) is/are also 
trapped inside the $\mathrm{C}_{60}$ cage. The presence of $\mathrm{W}$ atoms has dramatically increased the nucleation rate of the onion-like structures. The pentagonal carbon rings are the source for the spiral growth. Polyhedronshape spiral shells have been observed, and the diameter of the innermost layer is approximately $0.7 \mathrm{~nm}$. These spiral shells suggest a model for the growth of large fullerenes and carbon spheres. Experimental data suggest the existence of singleshell toroidal fullerenes. The height of the toroidal fullerenes can be as small as $0.4 \mathrm{~nm}$, in agreement with the theoretical prediction.

Acknowledgements - Thanks to the referee and editor for critical comments and many useful suggestions.

\section{REFERENCES}

I. Smith, W. R., in Encyclopedia of Chemical Technology, Vol. 3. Interscience, New York, 1949. pp. 39-43 and pp. $130-144$.

2. Studebaker, M. L., Rub. Chem. and Tech., 1957, 30, 1401.

3. Kroto, H. W., Heath, J. R., O'Brien, S. C., Curl, R. F. and Smalley, R. E., Nature, 1985, 318, 162

4. Kroto, II. W. and McKay, K., Nature, 1988, 331, 328.

5. Lamb, L. D., Huffman, D. R., Workman, R. K., Howells, S., Chen, T., Sarid, D. and Ziolo, R. F., Science, 1992, 255, 1413.

6. Iijima, S., Nature, 1991, 354, 56.

7. Wang, X. K., Lin, X. W., Song, S. N.. Dravid, V. P., Ketterson, J. B. and Chang, R. P. H., Carbon, 1995, 33, 949.

8. Amelinckx, S., Zhang, X. B., Bernaerts, D., Zhang, X. F., Ivanov, V. and Nagy, J. B., Science, 1994, 265, 635

9. Iijima, S., J. Crystal Growth, $1980,50,675$.

10. Ugarte, D., Nature, 1992, 359, 707.
11. de Ileer, W. and Ugarte, D., Chem. Phys. Lett., 1993, 207, 480

12. Kuznetsov, V. L., Chuvilin, A. L., Butenko, Y. V., Mal'kov, I. Y. and Titov, M., Chem. Phys. Lett., 1994. 222, 343

13. Inagaki, M., Kuroda, K. and Sakai, M., Carbon, 1983. 21, 231.

14. Yamada, K. and Tobisawa, S., Carbon, 1989, 27, 845.

15. Z. L. Wang and Z. C. Kang, J. Phys. Chem., 1996 100,17725 .

16. Kang, Z. C. and Wang, Z. L., Phil. Mag. B, Philos. Maga. B, 1996, 73, 905 .

17. Wang, Z. L. and Kang, Z. C., Phil. Maga. B. Philos. Maga. $B, 1996,74,51$.

18. Kang, Z. C. and Wang, Z. L., J. Phys. Chem. 1996. $100,5163$.

19. Heckman, F. A.. Rubber Chem. and Technol. 1964. 37, No. 5.

20. Heidenreich, R. D., Hess, W. M. and Ban, L. L., J. Appl. Cryst., 1968, 1, 1

21. Iijima, S., Ichihashi, T. and Ando, Y., Nature, 1992, 356, 776.

22. Kuznetsov, V. L., Chuvilin, A. L., Moroz, E. M., Kolomichuk, V. N., Shaikhutdinov, Sh. K. and Butenko, Yu. V., Carbon, 1994, 32, 873.

23. Feibelman, P. J. and Knotek, M. L.. Phvs. Rev. 1978 18,6531

24. Iijima, S., Micron, 1977, 8, 41 .

25. Cowley, J. M., in High Resolution Transmission Electron Microscopy, Ch. 1-3, eds P. Buseck, J. M. Cowley and L. Eyring. Oxford University Press, 1989

26. Saito, R., Dresselhaus, G. and Dresselhaus, M. S. Chem. Phys. Lett. 1992, 195, 537.

27. Brabec, C. J., Maiti, A. and Bernhole, J., Chem. Phys Lett., 1994, 219, 473.

28. Ihara, S. and Itoh, S., Carbon, 1995, 33, 931

29. Dresselhaus, M. S., Dresselhaus, G. and Saito, R., Carbon, 1995, 33, 883 .

30. Iijima, S. and Ichihashi, T., Nature (London), 1993, 363, 603 .

31. Bethune, D. S., Kiang, C. H., de Vries, M. S. Gorman, G., Savoy, R., Vazquez, J. and Beyers, R. Nature (London). 1993, 363, 605. 